\title{
Pathogenic Neisseria meningitidis utilizes CD147 for vascular colonization
}

\author{
Sandra C Bernard ${ }^{1-4,18}$, Nandi Simpson ${ }^{1-3,17,18}$, Olivier Join-Lambert ${ }^{5-8}$, Christian Federici ${ }^{1-3}$, \\ Marie-Pierre Laran-Chich ${ }^{1-3}$, Nawal Maïssa ${ }^{1-3}$, Haniaa Bouzinba-Ségard ${ }^{1-3}$, Philippe C Morand ${ }^{1,2,7}$, \\ Fabrice Chretien ${ }^{9-11}$, Saïd Taouji ${ }^{12}$, Eric Chevet ${ }^{12}$, Sébastien Janel ${ }^{13-16}$, Frank Lafont ${ }^{13-16}$, Mathieu Coureuil ${ }^{5-7}$, \\ Audrey Segura ${ }^{5-7}$, Florence Niedergang ${ }^{1-3}$, Stefano Marullo ${ }^{1-3}$, Pierre-Olivier Couraud ${ }^{1-3}$, Xavier Nassif ${ }^{5-8}$ \& \\ Sandrine Bourdoulous ${ }^{1-3}$
}

\begin{abstract}
Neisseria meningitidis is a cause of meningitis epidemics worldwide and of rapidly progressing fatal septic shock. A crucial step in the pathogenesis of invasive meningococcal infections is the adhesion of bloodborne meningococci to both peripheral and brain endothelia, leading to major vascular dysfunction. Initial adhesion of pathogenic strains to endothelial cells relies on meningococcal type IV pili, but the endothelial receptor for bacterial adhesion remains unknown. Here, we report that the immunoglobulin superfamily member CD147 (also called extracellular matrix metalloproteinase inducer (EMMPRIN) or Basigin) is a critical host receptor for the meningococcal pilus components PilE and PilV. Interfering with this interaction potently inhibited the primary attachment of meningococci to human endothelial cells in vitro and prevented colonization of vessels in human brain tissue explants ex vivo and in humanized mice in vivo. These findings establish the molecular events by which meningococci target human endothelia, and they open new perspectives for treatment and prevention of meningococcus-induced vascular dysfunctions.
\end{abstract}

N. meningitidis, also referred to as meningococcus, is an obligate human Gram-negative bacterium that normally resides in the nasopharyngeal mucosa without affecting the host, a phenomenon known as carriage ${ }^{1}$. Pathology is initiated when meningococci gain access to the bloodstream, multiply in the blood and disseminate into various tissues, causing meningitis or purpura fulminans, the most severe form of meningococcal invasive disease. Despite antimicrobial therapy, systemic meningococcal infections remain a major cause of mortality and severe neurological sequelae ${ }^{2-4}$. The endothelium lining blood and lymphatic vessels is a key barrier separating circulating body fluids from host tissues and is a major target for several pathogenic bacteria ${ }^{5}$. A crucial step in the pathophysiology of invasive bloodborne meningococci is their adhesion to and proliferation in both peripheral and brain blood microvessels, a process referred to as vascular colonization ${ }^{6}$. This intimate interaction of meningococci with endothelial cells leads to deregulated inflammatory and coagulation processes, endothelial dysfunction and, ultimately, the breach of endothelial barriers and bacterial dissemination into perivascular tissues ${ }^{4}$. Deciphering the mechanisms that govern this initial pathophysiological step of invasive meningococcal diseases represents an essential development toward the design of new therapeutic approaches to control bacterial infection and subsequent vascular and tissue damage. The capacity of pathogenic capsulated meningococci to interact tightly with human endothelial cells relies on the expression of type IV pili ${ }^{3,5}$. However, the host cell adhesion receptor that mediates the bacterial attachment to human vessels has remained unknown.

Type IV pili are long filamentous structures that extend from the bacterial cell surface. They mediate adhesion to endothelial cells and signaling events that eventually lead to bacterial translocation through endothelia ${ }^{7-10}$. These structures are heteromultimeric assemblies of pilin subunits that form helical fibers ${ }^{11}$. The major pilin subunit, Pile, constitutes the essential fiber scaffold and is subject to antigenic variation. Other less abundant ('minor') pilins, such as PilV, PilX and ComP, structurally resemble PilE and participate in various pilus-related functions such as adhesion, bacterial aggregation and DNA uptake, respectively ${ }^{12-14}$. Both PilE and PilV have been shown to be involved in adhesion to host cells $9,15-18$. Furthermore, these two pilins were recently reported to activate the $G$ protein-coupled $\beta_{2}$-adrenergic receptor $\left(\beta_{2}-\mathrm{AR}\right)$, which promotes the endothelial signaling events that are subsequent to bacterial adhesion ${ }^{9,19}$. However, $\beta_{2}$-AR-depleted endothelial cells, although unable to promote signaling events, still support pilus-mediated bacterial adhesion,

${ }^{1}$ INSERM, U1016, Institut Cochin, Paris, France. ${ }^{2}$ CNRS, UMR8104, Paris, France. ${ }^{3}$ Université Paris Descartes, Sorbonne Paris Cité, Paris, France. ${ }^{4}$ Université Paris Diderot, Paris, France. ${ }^{5}$ INSERM, U1151, Institut Necker Enfants Malades, Paris, France. ${ }^{6}$ CNRS, UMR8253, Paris, France. ${ }^{7}$ Faculté de Médecine, Université Paris Descartes, Sorbonne Paris Cité, Paris, France. ${ }^{8}$ Assistance Publique-Hôpitaux de Paris, Hôpital Necker Enfants Malades, Paris, France. 9 Unité Histopathologie Humaine et Modèles Animaux, Institut Pasteur, Paris, France. 10Faculté de Médecine, Université Versailles Saint-Quentin-en-Yvelines, Versailles, France. ${ }^{11}$ Assistance Publique-Hôpitaux de Paris, Hôpital Raymond Poincaré, Paris, France. ${ }^{12}$ BMYscreen, INSERM U1053, Université de Bordeaux, Bordeaux, France. ${ }^{13}$ Cellular Microbiology of Infectious Pathogens, Center for Infection and Immunity of Lille, Institut Pasteur de Lille, Lille, France. ${ }^{14}$ CNRS UM8204, Lille, France. 15INSERM U1019, Lille, France. 16Biolmaging Center Lille (BICeL), Université Lille Nord de France, Lille, France.

17Present address: Research Department of Infection, University College London, London, UK. 18These authors contributed equally to this work. Correspondence should be addressed to S.B. (sandrine.bourdoulous@inserm.fr). 
thus indicating that the primary meningococcal attachment requires another, yet unidentified, host receptor for type IV pili.

Here, we report that CD147, a member of the immunoglobulin (Ig) superfamily, is a receptor for type IV pilus-mediated adhesion of pathogenic meningococci to human brain or peripheral endothelial cells through interaction with both major and minor pilins-PilE and PilV-and we establish the central role of CD147 for vascular colonization by meningococci in vivo.

\section{RESULTS}

\section{CD147 is a candidate endothelial receptor for meningococci}

To identify candidate receptors for the initial attachment of piliated, capsulated meningococci, we used a gain-of-function strategy in BB19 cells, a dedifferentiated human brain endothelial cell line transformed by papilloma virus that is poorly permissive to meningococcal adhesion under basal conditions. Treatment of BB19 cells with phorbol ester 12-O-tetradecanoylphorbol-13-acetate (TPA), a potent transcriptional regulator, induced enhanced adhesion of the meningococcus strain Nm2C4.3 (Supplementary Fig. 1a). Treatment with the transcription inhibitor actinomycin D blocked the TPA effect, suggesting the regulated expression of a putative receptor by TPA at the transcriptional level (Supplementary Fig. 1a). As expected for type IV pilusmediated adhesion, a nonpiliated meningococcal PilE-null mutant ( $\triangle$ pilE) failed to adhere to TPA-treated cells (Supplementary Fig. 1b). In order to identify upregulated genes in permissive cells, we used a differential, quantitative, large-scale analysis of gene expression (Online Methods) that showed 211 genes upregulated by TPA among 10,478 analyzed genes. Six of them encoded membrane-associated proteins (Supplementary Table 1). Among those, four proteins had either limited tissue distribution (sialic acid-binding Ig-type lectin 4 (siglec 4), siglec 11 and delta- and Notch-like epidermal growth factor-related receptor were located mainly in the brain) or limited surface exposure (phosphatidylethanolamine-binding protein). Therefore, we instead focused on the type 1 plasma membrane protein CD147, a member of the

a $\mathrm{hCMEC/D3}$

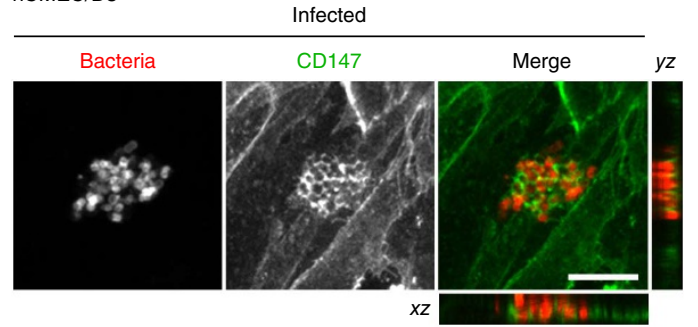

Ig superfamily ${ }^{20}$ and a well-established marker of brain capillaries ${ }^{21,22}$, and CD59, a glycosylphosphatidylinositol-anchored glycoprotein known to inhibit membrane attack complex formation in the complement cascade and to induce signaling events ${ }^{23}$. We confirmed that TPA treatment of BB19 cells upregulated expression of CD147 and CD59 (Supplementary Fig. 1c). CD147 expression was enriched at sites of meningococcal adhesion, whereas CD59 was not (Supplementary Fig. 1d).

We investigated the relevance of CD147 and CD59 as endothelial candidate receptors for meningococcal adhesion using two fully differentiated human endothelial cell lines isolated from either brain (hCMEC/D3) ${ }^{24}$ or bone marrow (human bone marrow endothelial cell, HBMEC $)^{25}$ capillaries. As observed on TPA-treated BB19 cells, CD59 was not recruited to sites of meningococcal adhesion on HBMECs (Supplementary Fig. 2a). In noninfected fully polarized hCMEC/D3 or HBMEC monolayers, CD147 was enriched at cell-cell junctions, where it is engaged in homophilic interactions ${ }^{26,27}$, and was also present at the luminal surface (Fig. 1a and Supplementary Fig. 2a). Upon infection with Nm2C4.3, CD147 accumulated at sites of meningococcal adhesion to hCMEC/D3 cells (Fig. 1a) and HBMECs (Supplementary Fig. 2a), as already observed for the signaling $\beta_{2}$-AR receptor ${ }^{9,19}$. CD147 accumulation occurred rapidly upon contact of diplococci with the cell surface (Fig. 1b) and was independent of the recruitment of the $\beta_{2}-A R$, as expected for a receptor mediating initial adhesion (Fig. 1c,d and Supplementary Fig. 3). CD147 thus seemed to be a potential endothelial receptor candidate for $N$. meningitidis.

\section{CD147 allows meningococcal adhesion to endothelial cells}

To establish the role of CD147 in meningococcal adhesion, we transfected endothelial cells with CD147-specific siRNAs. The reduction of CD147 surface expression in hCMEC/D3 cells and HBMECs (75\% and $80 \%$, respectively) correlated with decreased meningococcal adhesion to both cerebral or peripheral endothelial cells (65\% and $75 \%$, respectively; Fig. 2a,b). These data were supported by the rescue of meningococcal adhesion in CD147-complemented endothelial cells (Fig. 2b).

b

Figure 1 CD147 accumulates at sites of meningococcal adhesion independently of $\beta_{2}$-AR activation. (a) CD147 staining in noninfected and infected polarized monolayer of hCMEC/D3 cells. CD147 (green) and meningococcal colonies (red) in reconstructed images of 2-h infection; image reconstructions along $x z$ and $y z$ axes are shown. Scale bars, $10 \mu \mathrm{m}$. (b) Magnification of an adherent diplococcus $10 \mathrm{~min}$ after infection. Scale bar, $5 \mu \mathrm{m}$. (c) $\mathrm{hCMEC/D3}$ cells expressing exogenous YFP-tagged $\beta_{2}-A R\left(\beta_{2}-A R-Y F P\right)$ left untreated (NT) or preincubated for $1 \mathrm{~h}$ with $10 \mu \mathrm{M}$ isoproterenol (ISO) to induce endocytosis of the $\beta_{2}$-AR before 2 -h infection with wild-type meningococci. Arrows indicate the location of adherent meningococcus colonies. Scale bars, $10 \mu \mathrm{m}$. Images are representative of 3 independent experiments. (d) Percentage of the bacterial colonies associated with ezrin, CD44 or CD147 recruitment to sites of meningococcal adhesion in untreated cells or after cell pretreatment with $10 \mu \mathrm{M}$ isoproterenol to promote $\beta_{2}$-AR endocytosis. Mean \pm s.e.m.; $n=3$ experiments; ${ }^{* *} P<0.001$, two-way analysis of variance (ANOVA).

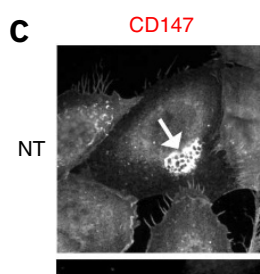
Noninfected
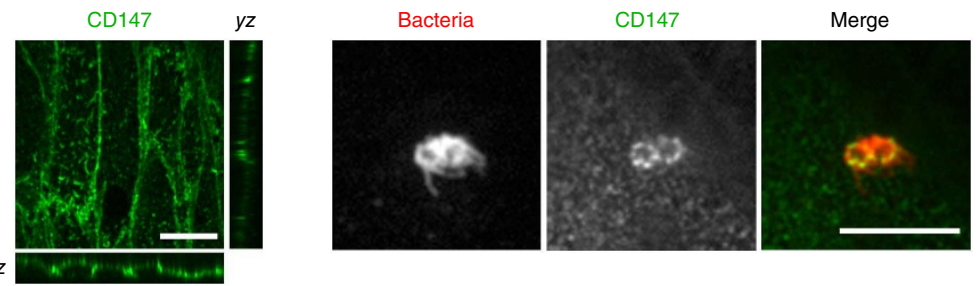

ISO
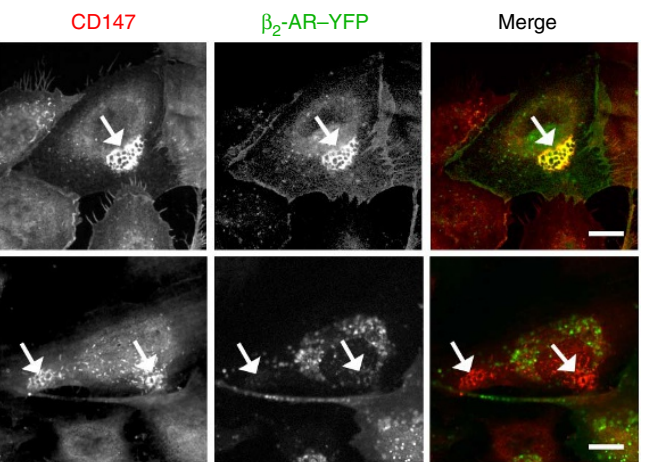

d

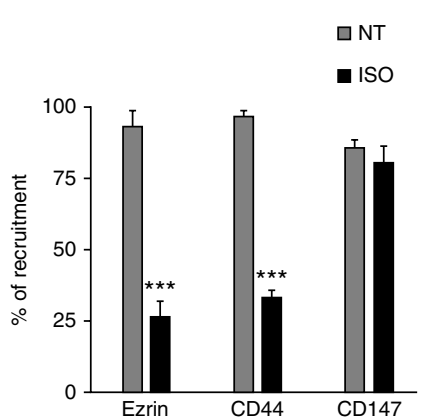



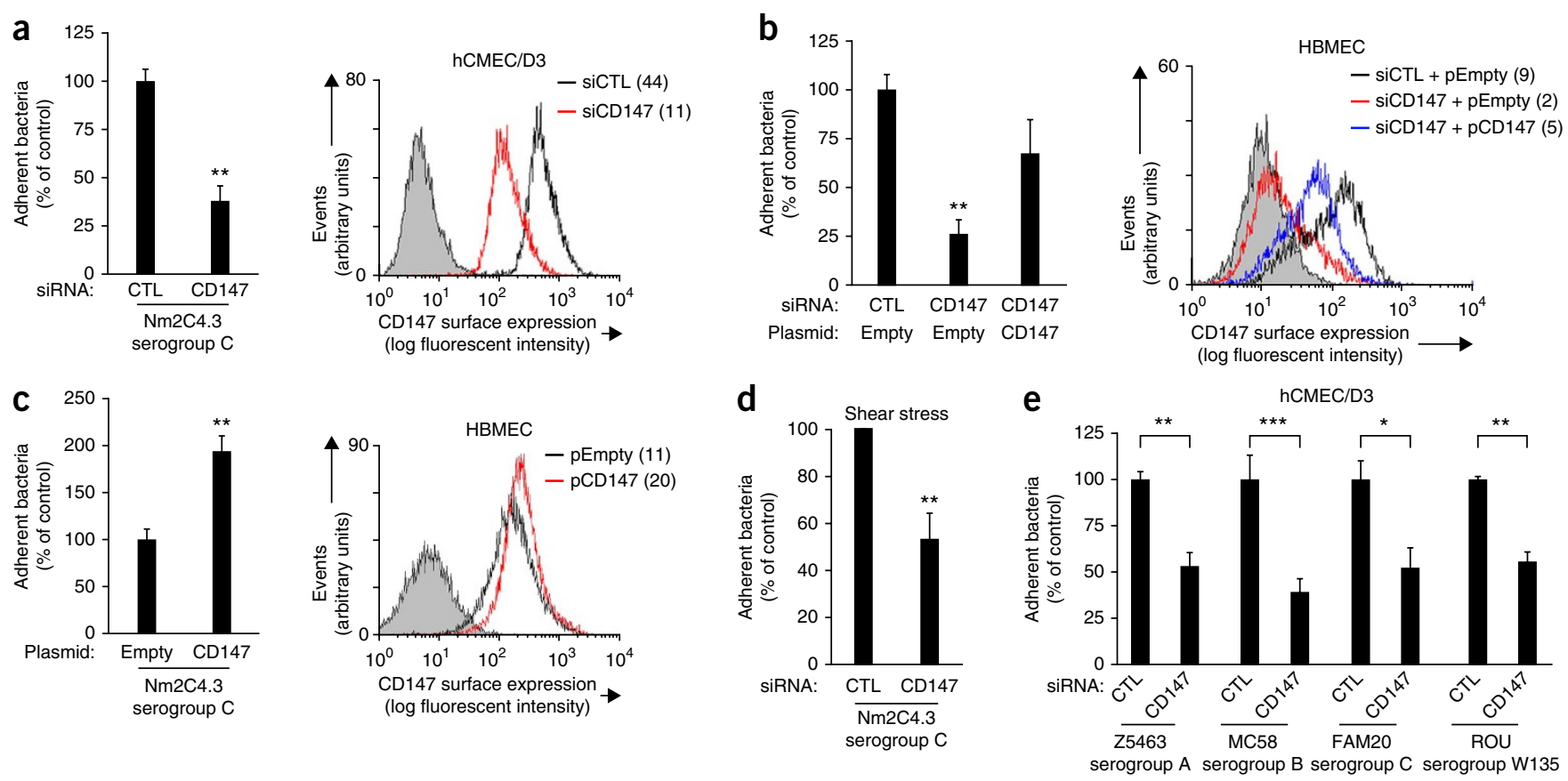

Figure 2 CD147 depletion, soluble CD147 and anti-CD147 antibodies inhibit $N$. meningitidis adhesion to human endothelial cells.

(a) Left, adhesion of Nm2C4.3 to CD147-depleted (siRNA CD147) or control (siRNA CTL) hCMEC/D3 cells, quantified following a 30-min infection. Right, FACS analysis of CD147 expression in hCMEC/D3 cells, with isotype control as shaded area and mean fluorescence intensities in parentheses. Mean \pm s.e.m., $n=4$; $* * P<0.05$, two-tailed Student's
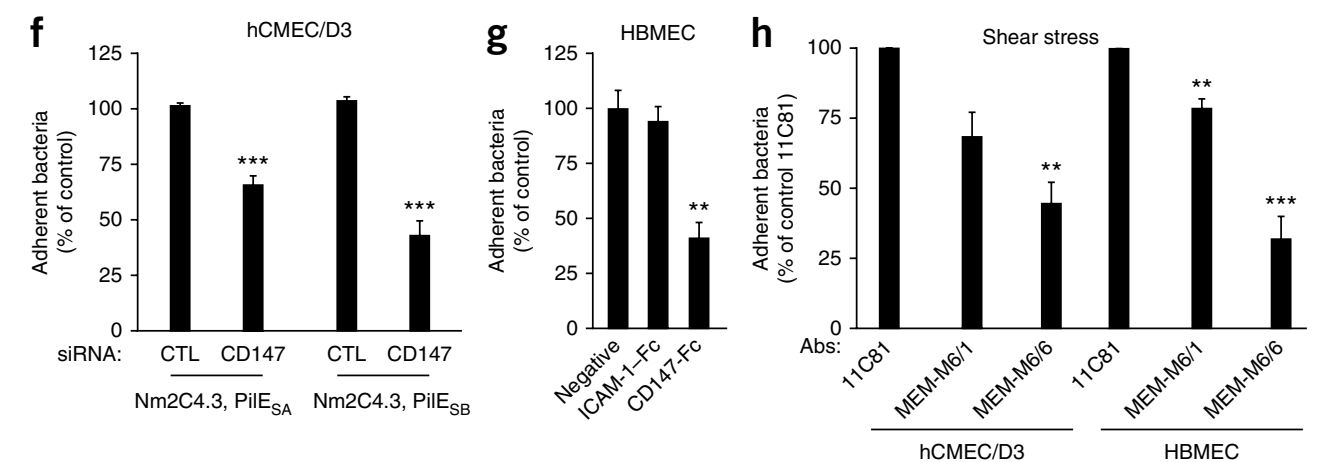

$t$-test. (b) Left, adhesion of Nm2C4.3 to

control hBMEC cells (siRNA CTL + empty vector), CD147-depleted (siRNA CD147 + empty vector) or CD147-complemented HBMECs (siRNA CD147 + plasmid encoding CD147), quantified following a 30-min infection. Right, FACS analysis of CD147 expression in HBMEC cells, with isotype control as shaded area and mean fluorescence intensities in parentheses. ${ }^{*} P=0.0025$, two-tailed Student's $t$-test. (c) Left, adhesion of Nm2C4.3 to control (empty plasmid) or CD147-overexpressing (CD147 plasmid) hBMEC cells, quantified following a 30-min infection. Right, FACS analysis of CD147 expression in HBMEC cells, with isotype control as shaded area and mean fluorescence intensities in parentheses. Mean \pm s.e.m., $n=3$; ${ }^{* *} P=0.0094$, twotailed Student's $t$-test. (d) Adhesion of Nm2C4.3 to CD147-depleted or control hCMEC/D3 cells, quantified following a 10-min infection under shear stress (0.04 dynes $/ \mathrm{cm}^{2}$ ). ${ }^{* *} P<0.01$, two-tailed Student's $t$-test. (e) Adhesion of meningococcus strains belonging to different sequence types and capsular serotypes to CD147-depleted or control hCMEC/D3 cells, quantified following a 30-min infection. Mean \pm s.e.m., $n=4 ;{ }^{*} P=0.025,{ }^{* *} P=0.001,{ }^{* * *} P=0.0008$, twotailed Student's $t$-test. (f) Adhesion of Nm2C4.3 pilin variant strains expressing PilE $E_{S A}$ or Pil $E_{S B}$ to CD147-depleted or control hCMEC/D3 cells, quantified following a 30-min infection. Mean \pm s.e.m., $n=6$; ${ }^{* * *} P<0.001$, two-way ANOVA. (g) Adhesion of Nm2C4.3 to HBMECs in the absence (negative) or in the presence of purified CD147-Fc or ICAM-1-Fc (both at $5 \mu \mathrm{g} \mathrm{ml}-1$ ), quantified following a 30-min infection. ** $P<0.01$, one-way ANOVA. (h) Adhesion of Nm2C4.3 to HBMECs and hCMEC/D3 cells preincubated with $10 \mu \mathrm{g} \mathrm{ml}-1$ of antibodies targeting ICAM-1 (11C81), the N-terminal Ig domain of CD147 (MEM-M6/1) or the C-terminal Ig domain of CD147 (MEM-M6/6), quantified following a 10-min infection under shear stress $(0.04$ dynes/cm²).

Abs, antibodies. Mean \pm s.e.m., $n=3 ;{ }^{* *} P<0.01,{ }^{* *} P<0.001$, one-way ANOVA.

Conversely, increased expression of CD147 on HBMECs increased bacterial adherence (Fig. 2c). This was specific to CD147, as depletion of CD59 did not affect bacterial adhesion to HBMECs (Supplementary Fig. 2b). Furthermore, depletion of CD44 or intercellular adhesion molecule-1 (ICAM-1), two membrane-associated molecules recruited to sites of bacterial adhesion upon type IV pilus-mediated signaling events $^{7}$, did not affect meningococcal adhesion to either hCMEC/D3 cells or HBMECs (Supplementary Fig. 4).

With the aim of approaching the physiological conditions of shear stress that are encountered in human blood microvasculature, we infected endothelial cells with meningococci in controlled laminar flow chambers that enable the quantification of initial bacterial contacts with the endothelial cell surface ${ }^{28}$. We observed that attenuation of CD147 expression by $65 \%$ on hCMEC/D3 cells reduced the number of initial adhesion events by $50 \%$ (Fig. 2d), confirming, under dynamic conditions, the selective effect of CD147 depletion on meningococcal adhesion.

Pathogenic meningococci belong to different capsular groups and show great capacity for antigenic variation in the pilE gene ${ }^{29}$ with no alteration in type IV pilus-mediated primary adhesion to host cells ${ }^{28,30}$. We therefore performed adhesion experiments using meningococcal strains belonging to different capsular serogroups (Z5463, serogroup A; MC58, serogroup B; FAM20, serogroup C; and ROU, serogroup W135) (Fig. 2e), as well as derivatives of $\mathrm{Nm} 2 \mathrm{C} 4.3$ (serogroup C) displaying different variants of the major pilin subunit 

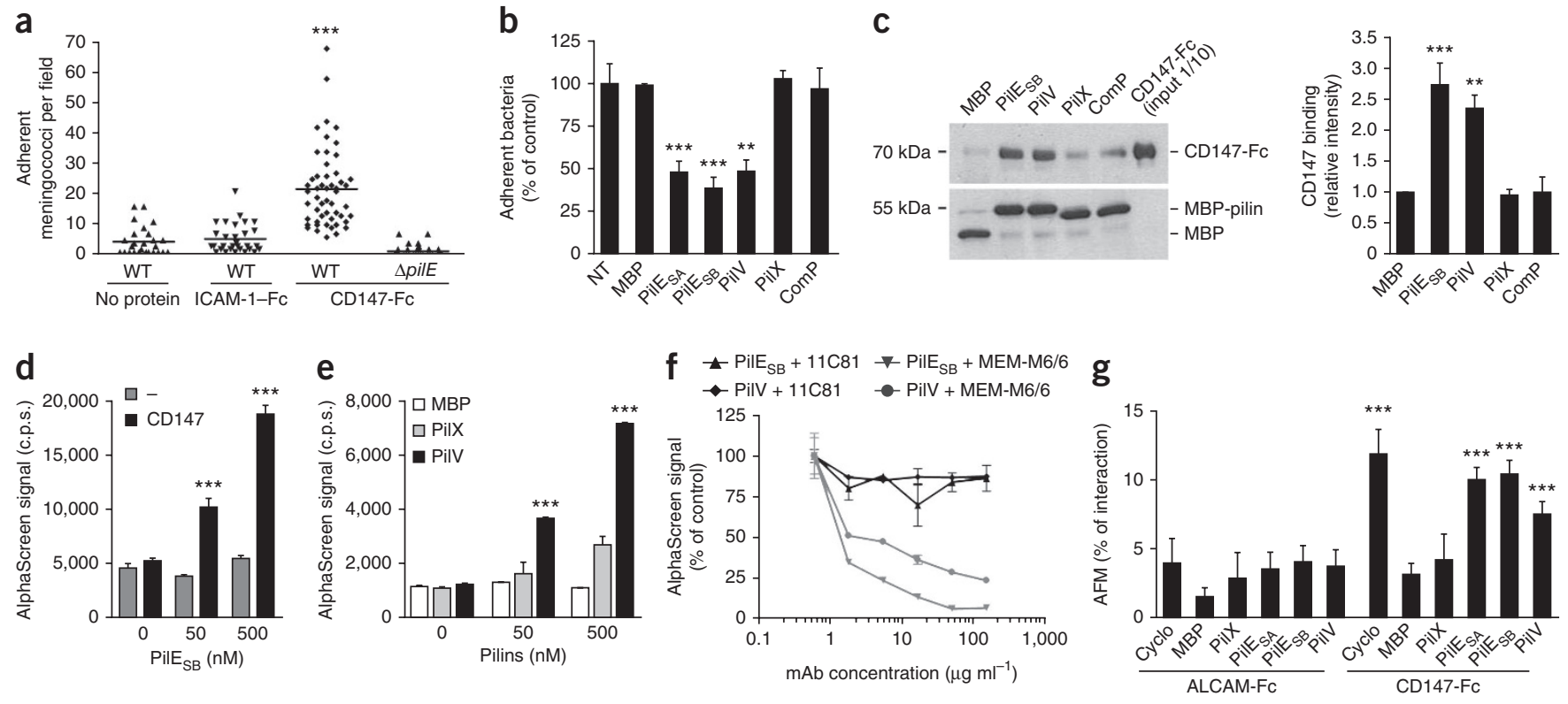

Figure 3 The major pilin PilE and the minor pilin PilV interact directly with CD147. (a) Adhesion of wild-type (WT) and nonpiliated ( $\Delta$ pilE, as control) meningococci to immobilized CD147-Fc, or to ICAM-1-Fc or no immobilized protein as controls, quantified following a 1-h infection. Mean \pm s.e.m., $n=4 ;{ }^{* *} P<0.001$, one-way ANOVA. (b) Adhesion of Nm2C4.3 to hCMEC/D3 cells in the absence (Nontreated, NT) or in the presence of soluble MBP-pilins (PilE $E_{S A}, P i l E_{S B}$ PilV, PilX or ComP) or MBP as a control, quantified following a 30-min infection. Mean \pm s.e.m., $n=4 ; * *<0.01$, $* * * P<0.001$, one-way ANOVA. (c) Left, CD147-Fc coprecipitation with staphylococci conjugated with the indicated MBP-pilins, or MBP alone as a control. Right, quantification of CD147 immunoprecipitation. Mean \pm s.e.m., $n=4 ; * * P<0.01$, ${ }^{* * *} P<0.001$, one-way ANOVA. (d) AlphaScreen analysis of the interaction between CD147-FC (500 nM) or vehicle ( $)$ ) as a control, and increasing MBP-PilE $\mathrm{SB}$ concentrations. Mean \pm s.e.m., $n=2$; ${ }^{* * * P}<0.001$, two-way ANOVA. (e) AlphaScreen analysis of the interaction between CD147-FC (500 nM) and MBP, MBP-PiIX or MBP-PilV at various concentrations. Mean \pm s.e.m., $n=2$ experiments; ${ }^{* *} P<0.001$, two-way ANOVA. (f) AlphaScreen analysis of the interaction between CD147-Fc $(100 \mathrm{nM})$ and MBP-PilE $\mathrm{SB}$ or MBP-PilV (500 nM) in the presence of increasing concentration of the anti-CD147 antibody (MEM-M6/6) or anti-ICAM-1 (11C81, as control). mAb, monoclonal antibody. (g) AFM analysis of the retraction forces between cantilever tips functionalized with MBP, MBP-pilins (PiIX, PilV, PilE SA and PilE $_{S B}$ ) or cyclophilin (Cyclo, positive control) and CD147-Fc or activated leukocyte-cell adhesion molecule-1 (ALCAM-1)-FC (control). Bar plots indicate the percentage of force curves with interaction events. Mean \pm s.e.m.; $n=7$. ${ }^{* *} P<0.001$, one-way ANOVA.

PilE, designated PilE $\mathrm{SA}_{\mathrm{A}}$ and $\mathrm{PilE}_{\mathrm{SB}}$ (Fig. 2f). Reduction of CD147 expression affected the adhesion of these different strains and variants similarly, demonstrating that the capacity of CD147 to support type IV pilus-dependent adhesion was independent of capsular antigen and of antigenic variation of PilE.

Competition experiments with CD147-Fc, a soluble recombinant chimeric molecule containing the extracellular domain of CD147 fused to the Fc domain of human IgG1, showed that CD147-Fc caused a $50 \%$ reduction in bacterial adhesion to HBMEC cells, whereas soluble ICAM-1-Fc had no effect (Fig. 2g). To confirm that the extracellular portion of CD147 is a binding site for meningococci, we assayed two antibodies specific for CD147, MEM-M6/1 and MEM-M6/6, which respectively bind to the extracellular $\mathrm{N}$-terminal and the $\mathrm{C}$-terminal Ig domains of CD147, as interaction inhibitors (Supplementary Fig. 5a). Both antibodies labeled noninfected hCMEC/D3 cells similarly (Supplementary Fig. 5b), but only MEM-M6/1 efficiently labeled CD147 molecules recruited to bacterial adhesion sites, suggesting that the MEM-M6/6 antibody and meningococci compete for the same binding site in the C-terminal Ig domain of CD147 (Supplementary Fig. 5c). Accordingly, preincubation of hCMEC/D3 cells or HBMECs with the MEM-M6/6 antibody before infection inhibited the initial attachment of $\mathrm{Nm} 2 \mathrm{C} 4.3$ under shear stress by $55 \%$ and $75 \%$, respectively, whereas the MEM-M6/1 antibody reduced the initial attachment by only $25 \%$ and $20 \%$ (Fig. 2 h). Overall, our data indicate that CD147 is a critical receptor for the primary attachment of piliated meningococci to both peripheral and brain human endothelial cells.

\section{Meningococcal pilins PilE and PilV interact with CD147}

We next investigated whether the interaction of meningococcus with CD147 was direct by performing meningococcal adhesion experiments using recombinant CD147-Fc immobilized on glass coverslips. Meningococci adhered in a type IV pilus-dependent manner to immobilized CD147-Fc but not to ICAM-1-Fc or noncoated control wells (Fig. 3a). To identify the bacterial pilus components involved in interaction with CD147, we used Escherichia coli to produce the minor pilins PilV, PilX and ComP, as well as two variants of the major pilin PilE ( $\mathrm{PilE}_{\mathrm{SA}}$ and $\mathrm{PilE}_{\mathrm{SB}}$ ), as fusion proteins with maltose-binding protein (MBP). Both PilE variants and PilV fusion proteins reduced the adhesion of piliated, capsulated meningococci by $50-60 \%$ in competition assays, whereas PilX or ComP had no effect (Fig. 3b).

We further assessed the direct interaction of CD147 with these pilins using several complementary approaches. First, purified $\mathrm{PilE}_{\mathrm{SB}}$ or PilV recombinant pilins immobilized on inactivated staphylococci could selectively pull down soluble CD147-Fc (Fig. 3c). Second, in a protein-protein interaction assay based on oxygen singlet transfer (AlphaScreen), we detected a dose-dependent association with CD147-Fc for both PilE $\mathrm{SB}_{\mathrm{SB}}$ and PilV, but not for the other minor pilins (Fig. 3d,e). This interaction was independent of antigenic variation in the PilE subunit (Supplementary Fig. 6a) and was specific to CD147 (Supplementary Fig. 6b). As observed above for meningococcal adhesion to human endothelial cells, these interactions were inhibited by the MEM-M6/6 antibody but not by the control anti-ICAM-1 antibody (11C81) (Fig. 3f and Supplementary Fig. 6c). 
Figure 4 The major pilin PilE and the minor pilin PilV are essential bacterial components for vascular colonization. (a) Adhesion of Nm2C4.3 wild-type strain or $\triangle$ pilE and $\triangle$ pilV mutant derivatives to hCMEC/D3 cells and HBMECs. Mean \pm s.e.m., $n=4$; ${ }^{* *} P<0.001$, one-way ANOVA. $(\mathbf{b}, \mathbf{c})$ Colonization of human vessels in grafted human skin by Nm2C4.3 wild-type strain or the $\Delta$ pilE or the $\Delta$ pilV mutant derivatives. (b) Mean \pm s.e.m. of the vascular colonization index. Analysis of 10 sections per graft, $n=6$ grafts per condition. ${ }^{* *} P<0.001$, one-way ANOVA.

(c) Representative fluorescence microscopy showing the different bacterial derivatives (red) associated with human vessels (basal lamina collagen IV, green) in the skin. Scale bars, $100 \mu \mathrm{m}$. Images are representative of 6 different human skin grafts.

Third, using surface plasmon resonance, we found a specific interaction of PilE $\mathrm{SB}^{-}$or PilV-coated staphylococci with immobilized CD147 (Supplementary Fig. 7), but we observed no interaction when we used monomeric pilins (data not shown). These results indicate that, despite relatively low affinities of $\mathrm{PilE}_{\mathrm{SB}}$ and PilV monomers for CD147, their clustering on staphylococci can provide sufficient avidity to CD147, which is consistent with the multimeric organization of pilins within pilus fibers. We then quantified the interaction of PilE variants or PilV with CD147 by atomic force microscopy (AFM) (Fig. 3g). We observed a load-dependent correlation for the interaction forces of PilE variants or PilV with CD147, further confirming the specificity of this interaction (data not shown). Measured interaction forces with CD147 $(62 \pm 15 \mathrm{pN}$, $58 \pm 16 \mathrm{pN}$ and $49 \pm 12 \mathrm{pN}$, for $\mathrm{PilE}_{\mathrm{SA}}, \mathrm{PilE}_{\mathrm{SB}}$ and PilV, respectively) were only about twofold lower than that between CD147 and its established ligand cyclophilin ${ }^{31}(128 \pm 35 \mathrm{pN})$. These data, consistent with a previously described role for PilE and PilV in Neisseria species pilus-mediated adhesion to host cells ${ }^{9,15-18}$, confirm the direct participation of PilE and PilV in the selective interaction of meningococci with CD147.

\section{Vascular colonization in vivo requires pilins PilE and PilV}

To establish the role of PilE and PilV in meningococcal adhesion to endothelial cells, we used defective strains of Nm2C4.3. Neither the nonpiliated $\triangle p i l E$ nor the piliated pilV-null $(\Delta p i l V)$ mutants were able to adhere in vitro to hCMEC/D3 or HBMEC cells (Fig. 4a). As a control, $\Delta p i l E$ and $\Delta p i l V$ complemented strains recovered bacterial adhesion to both endothelial cell lines with an efficiency correlated to the expression level of the complemented proteins (Supplementary Fig. 8a-c).
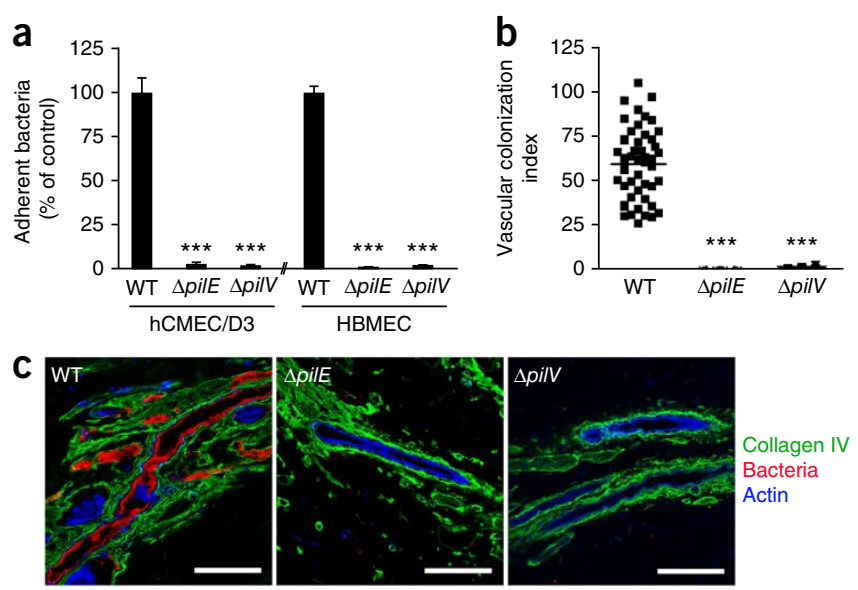

To further investigate the specific roles of PilE and PilV in endothelial colonization by meningococci in vivo and because this human specific pathogen does not colonize mouse vessels, we used a humanized mouse model of severe combined immunodeficiency (SCID) mice grafted with human skin, in which functional human blood vessels are maintained within the graft ${ }^{32,33}$. Although limited to dermal vessels, this model constitutes a unique tool to study meningococcal interaction with human endothelial cells under realistic conditions in a live host. As previously shown ${ }^{32}, 18 \mathrm{~h}$ after bacterial challenge, the Nm2C4.3 wild-type strain massively colonized the human dermal vasculature in a type IV pilus-dependent manner, as we observed no colonization with the nonpiliated $\Delta$ pilE derivative (Fig. 4 b,c). Consistent with our in vitro observation, the meningococcal $\Delta p i l V$ derivative did not associate with vessels in the grafted human skin (Fig. $4 \mathbf{b}, \mathbf{c}$ ), whereas both $\Delta$ pilE and $\Delta$ pilV complemented strains colonized human vessels with an efficiency correlating to the expression level of the complemented proteins (Supplementary Fig. 8d-f). These results establish PilE and PilV as essential bacterial components for in vivo type IV pilus-mediated vascular colonization.

\section{CD147 is required for in situ infection of brain vessels}

We further analyzed the selective interaction between meningococci and CD147 using an in situ meningococcal infection model of fresh
Figure 5 In situ interaction of $N$. meningitidis with human brain vessels requires both PilE and PilV expression. (a) Immunohistochemical analysis of bacteria (brown) in a whole human brain section infected with Nm2C4.3 wild-type strain. Arrows indicate bacterial aggregates within brain vessels in the cortical region. Scale bar, $1 \mathrm{~mm}$. (b) Detailed immunohistochemical analysis of the bacterial staining (blue) in the cortical region of serial human brain sections from the same donor infected with $\mathrm{Nm} 2 \mathrm{C} 4.3$ wild-type strain or the $\triangle$ pilE or the $\Delta$ pilV mutant derivatives. Scale bar, $100 \mu \mathrm{m}$.

(c) Detailed immunofluorescence analysis of bacteria (red), actin (green) and nucleus (blue) in the meningeal region (Virchow-Robin space) of serial human brain sections from the same donor infected with $\mathrm{Nm} 2 \mathrm{C} 4.3$ wild-type strain or the $\Delta$ pilE or the $\Delta$ pilV mutant derivatives. Scale bars, $100 \mu \mathrm{m}$.

(d) Quantification of adherent meningococci per 100- $\mu \mathrm{m}^{2}$ section, expressed as percentage of adherent wild-type bacteria. Mean \pm s.e.m., analysis of 3 brain sections per brain from 3 different donors; $* * * P<0.001$, one-way ANOVA. a
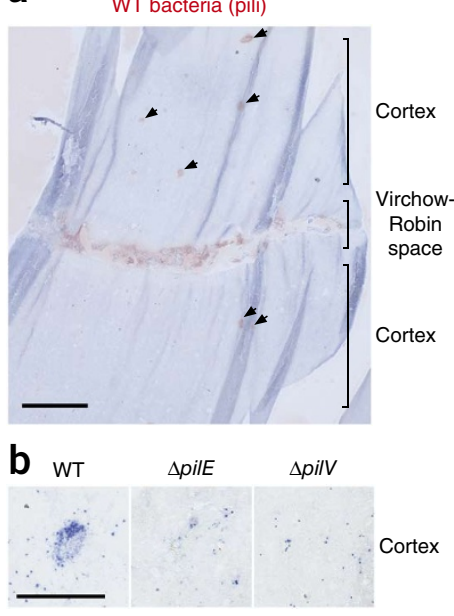

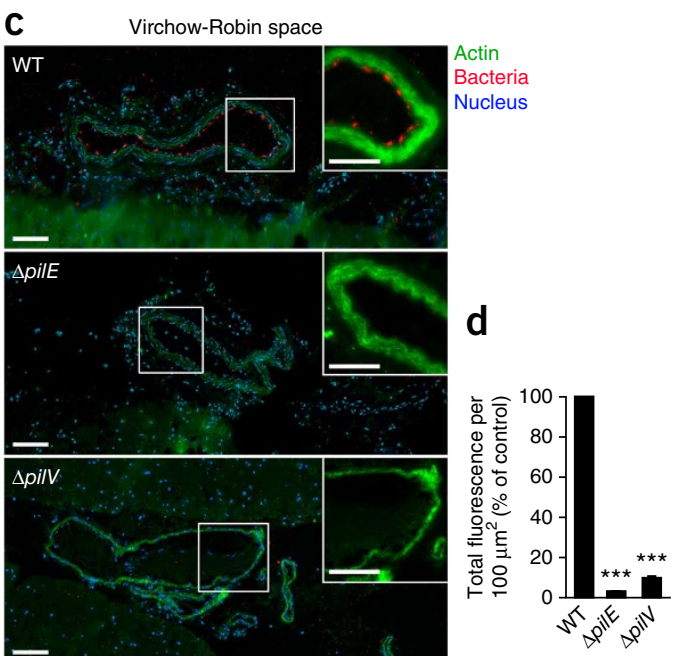


a

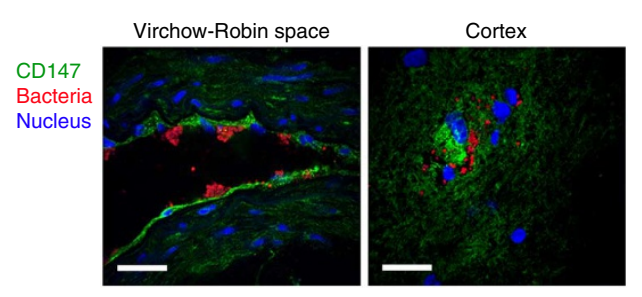

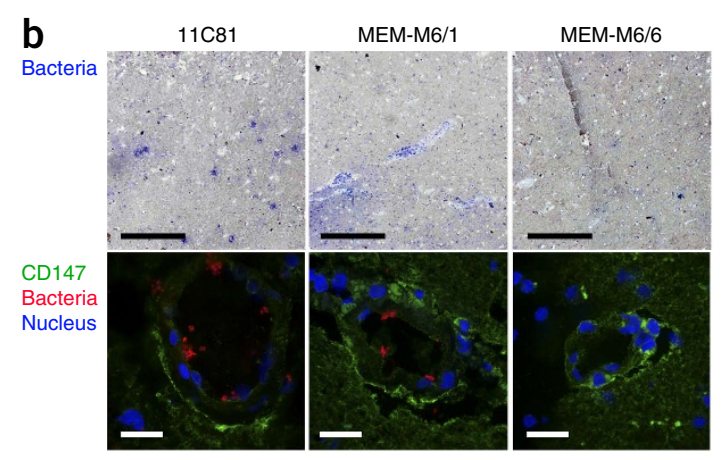

C

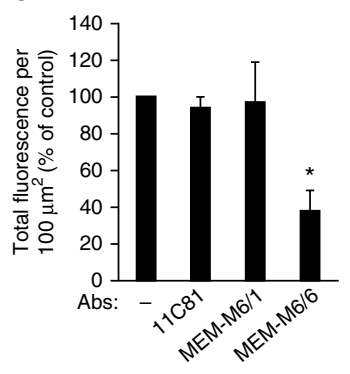

Figure 6 In situ infection of fresh human brain sections by N. meningitidis requires pilus interaction with CD147. (a) Immunofluorescence analysis of CD147 (green), meningococcus colonies (red) and nucleus (blue) in the Virchow-Robin space and cortical region of serial brain sections from the same donor infected with Nm2C4.3 wild-type strain for $1 \mathrm{~h}$. Scale bars, $20 \mu \mathrm{m}$. Images are representative of 6 different human skin grafts. (b) Immunohistochemical (top) and immunofluorescence (bottom) analysis of serial sections of cortical regions of the same donor, treated with $10 \mu \mathrm{g} \mathrm{m}^{-1}$ anti-ICAM-1 (11C81) or anti-CD147 (MEM-M6/1 and MEM-M6/6) antibodies before infection with Nm2C4.3 wild-type meningococci. Scale bars, $100 \mu \mathrm{m}$ (top) and $20 \mu \mathrm{m}$ (bottom). Images are representative of 3 different human skin grafts. (c) Mean \pm s.e.m. of total fluorescence per $100 \mu \mathrm{m}^{2}$ relative to fluorescence in absence of antibody, $n=4$ brain sections per donor from 3 different donors; * $P<0.05$, one-way ANOVA.

human frontal brain tissues obtained from deceased normal subjects, as, in this setting, histological and anatomical characteristics of the brain vessels are conserved. Meningococci incubated with tissue sections established specific tight association with brain vessels, predominantly in Virchow-Robin spaces and in cortical regions (Fig. 5a and Supplementary Fig. 9), reminiscent of neuropathological findings in patients with meningococcal meningitis ${ }^{28,34}$. Adhesion relied on the expression of both PilE and PilV, as $\Delta p i l E$ or $\Delta p i l V$ mutants adhered poorly (Fig. 5b-d and Supplementary Fig. 9).

Upon infection, wild-type meningococci developed microcolonies immediately adjacent to CD147-positive endothelial cells (Fig. 6a). We also found meningococci in the vicinity of CD147-positive leptomeningeal cells and of cortical brain vessels (Supplementary Fig. 10a,b), but they were not associated with glial or neuronal cells that do not express CD147 (Supplementary Fig. 10c), indicating a close correlation between meningococcal adhesion to fresh human brain tissue and CD147 expression. Consistent with in vitro cellular models, pretreatment of brain sections with the MEM-M6/6 antibody substantially reduced adhesion of meningococci to human brain vessels, whereas the MEM-M6/1 antibody or the control 11C81 anti-ICAM-1 antibody had no effect (Fig. 6b,c). Altogether, these data confirm that type IV pilus-mediated adhesion of meningococcus to CD147 is necessary for endothelial cell colonization by this bacterial pathogen.

\section{DISCUSSION}

This study demonstrates that the specific interaction between the meningococcal ligands PilE and PilV and the cellular host receptor CD147 is essential for meningococcal adhesion to human endothelial cells and colonization of human blood vessels, a prerequisite to the major vascular alterations that are the hallmark of invasive meningococcal infections. These results, which help unravel the molecular basis of a key pathophysiological step of meningococcal diseases, are consistent with previous reports suggesting that, in addition to its structural role in the pilus scaffold, the PilE subunit plays a direct role in bacterial adhesion to host cells ${ }^{15}$. PilV has also previously been shown to be required for the adhesion of the closely related Neisseria gonorrhoeae $^{18}$. Interaction with CD147 occurs independently of antigenic variation in the PilE subunit and of possible post-translational modifications. PilE and PilV have relatively low affinities for CD147, whereas multimeric pilins efficiently bind to CD147, indicating a system where avidity is crucial, consistent with the natural organization of pilins in multimeric complexes within pilus fibers. Because endothelial cells, and more particularly brain microvessels, express high levels of CD147, it is likely that CD147 expression level may also contribute to avidity, compensating for the weak affinity of the CD147-meningococcal pilins interaction. How these two pilus components act in concert to create the adhesive phenotype remains to be solved. Identification of the precise PilE and PilV epitopes required for interaction with CD147 will allow the development of targeted antibodies that could prevent type IV pilus-CD147 interaction, opening the path to new vaccine strategies against meningococcal infection.

CD147 is expressed on different cell types, such as erythrocytes and epithelial cells. Pilus-dependent interactions between meningococci and erythrocytes, causing hemagglutination, have been documented in vitro ${ }^{35,36}$, and meningococci have been described crossing polarized epithelia, indicating that meningococcus might interact with CD147 in a variety of cell types. Notably, several viruses, including HIV-1, severe acute respiratory syndrome coronavirus ${ }^{37}$ and measles virus $^{38}$, as well as the bacterial pathogen Listeria monocytogenes ${ }^{39}$, also target CD147 to adhere to and/or invade epithelial cells, indicating that CD147 constitutes an evolutionarily conserved efficient target for pathogens to infect tissues and spread within organisms. CD147 is also known as EMMPRIN, a master regulator of the production of matrix metalloproteinase $\mathrm{e}^{40}$. It is therefore likely that, in addition to its role in pathogen adhesion and invasion processes, CD147 also contributes to the disruption of the epithelial and endothelial barrier integrity by promoting local production of matrix metalloproteinases.

Whereas CD147 expression is required to support bacterial adhesion to endothelial cells of brain and peripheral origin, $\beta_{2}$-AR expression is necessary for meningococcus-triggered signaling ${ }^{8}$. $\beta_{2}$-AR is dispensable for adhesion ${ }^{9,19}$, but CD147-mediated meningococcal adhesion is a prerequisite for activating the $\beta_{2}$-AR- $\beta$-arrestin signaling pathway, thus suggesting that CD147 and $\beta_{2}$-AR might cooperate to strengthen meningococcal adhesion and promote subsequent activation of signaling events. Notably, a similar functional association of these two receptors has been reported in the context of erythrocyte infection by the human parasite Plasmodium falciparum, the agent of malaria, although the mechanism of this association is still unknown ${ }^{41,42}$. The implication of these two receptors in different tissues and in the context of different infectious diseases might reflect a preexisting functional connection between CD147 and $\beta_{2}$-AR that is hijacked by different pathogens to disseminate and induce multiple organ failure. 
Taken together, our data highlight a key role for CD147 in the cascade of pathophysiological events following meningococcal entry into the bloodstream. Agents or vaccines preventing type IV pilus interaction with CD147 might therefore be highly effective for treatment or prevention of meningococcal infection and associated vascular dysfunction.

\section{METHODS}

Methods and any associated references are available in the online version of the paper.

Note: Any Supplementary Information and Source Data files are available in the online version of the paper.

\section{ACKNOWLEDGMENTS}

We thank B.B. Weksler (Weill Cornell Medical College), J. Nelson (Oregon Health and Science University), M. Bukrinsky (George Washington University Medical Center) and P. Mangeat (Centre de Recherche en Biochimie Macromoléculaire) for providing HBMECs, BB19 cells, CD147 constructs and anti-ezrin antibody, respectively; P. Pelissier (Hôpital Saint Joseph) for providing human tissue samples for the human skin graft model; P. Bourdoncle, B. Durel, L. Stouvenel, M. Favier and C. Lessaffre for their technical help; J.M. Bruneau and F. Moreau for their technical help on surface plasmon resonance experiments; M. Popoff for his help on AFM analysis; Hamamatsu Photonics for providing access to their Nanozoomer scanning microscopy; and A. Fouet and J. Gavard for critical reading of the manuscript. S.C.B. was supported by a doctoral fellowship from Université Paris Diderot and Association pour la Recherche sur le Cancer. This work was supported by collaborative research grants from La Ville de Paris to S.B. and F.N., from the Agence Nationale de la Recherche of France (ANR-06-MIME-029; ANR-09-BLAN-0137-03) and the Université Paris Descartes (AAP-2011) to S.B., X.N. and S.M., from the Fondation pour la Recherche Médicale of France to X.N. and S.M. and from the ANR (ANR-10-EQPX-04-01) to F.L.

\section{AUTHOR CONTRIBUTIONS}

S.C.B., N.S., N.M. and H.B.-S. conducted the experiments; O.J.-L. conducted the in vivo experiments; C.F. and M.-P.L.-C. performed the serial analysis of gene expression adapted for downsized extracts experiment and analysis; P.C.M. prepared the complemented mutants and wrote the manuscript; M.C. and A.S. provided bacterial mutants; F.C. provided brain tissues and immunohistochemical analysis; S.T. and E.C. conducted the AlphaScreen assays; S.J. and F.L. conducted the AFM assays; P.-O.C. provided cell lines and discussed the results; F.N. provided reagents and discussed the results; S.M. and X.N. provided reagents and bacterial strains, wrote the manuscript and discussed the results; and S.B. designed the experiments, supervised the project and wrote the manuscript.

\section{COMPETING FINANCIAL INTERESTS}

The authors declare no competing financial interests.

Reprints and permissions information is available online at http://www.nature.com/ reprints/index.html.

1. Yazdankhah, S.P. \& Caugant, D.A. Neisseria meningitidis: an overview of the carriage state. J. Med. Microbiol. 53, 821-832 (2004).

2. Stephens, D.S., Greenwood, B. \& Brandtzaeg, P. Epidemic meningitis, meningococcaemia, and Neisseria meningitidis. Lancet 369, 2196-2210 (2007).

3. Virji, M. Pathogenic neisseriae: surface modulation, pathogenesis and infection control. Nat. Rev. Microbiol. 7, 274-286 (2009).

4. Brandtzaeg, P. \& van Deuren, M. Classification and pathogenesis of meningococcal infections. Methods Mol. Biol. 799, 21-35 (2012).

5. Lemichez, E., Lecuit, M., Nassif, X. \& Bourdoulous, S. Breaking the wall: targeting of the endothelium by pathogenic bacteria. Nat. Rev. Microbiol. 8, 93-104 (2010).

6. Melican, K. \& Dumenil, G. Vascular colonization by Neisseria meningitidis. Curr. Opin. Microbiol. 15, 50-56 (2012).

7. Doulet, N. et al. Neisseria meningitidis infection of human endothelial cells interferes with leukocyte transmigration by preventing the formation of endothelial docking structures. J. Cell Biol. 173, 627-637 (2006).

8. Coureuil, M. et al. Meningococcal type IV pili recruit the polarity complex to cross the brain endothelium. Science 325, 83-87 (2009).

9. Coureuil, M. et al. Meningococcus hijacks a $\beta_{2}$-adrenoceptor/ $\beta$-arrestin pathway to cross brain microvasculature endothelium. Cell 143, 1149-1160 (2010).

10. Dupin, N. et al. Chronic meningococcemia cutaneous lesions involve meningococcal perivascular invasion through the remodeling of endothelial barriers. Clin. Infect. Dis. 54, 1162-1165 (2012)

11. Craig, L. \& Li, J. Type IV pili: paradoxes in form and function. Curr. Opin. Struct. Biol. 18, 267-277 (2008)
12. Hélaine, S. et al. PilX, a pilus-associated protein essential for bacterial aggregation, is a key to pilus-facilitated attachment of Neisseria meningitidis to human cells. Mol. Microbiol. 55, 65-77 (2005).

13. Mikaty, G. et al. Extracellular bacterial pathogen induces host cell surface reorganization to resist shear stress. PLoS Pathog. 5, e1000314 (2009).

14. Brown, D.R., Helaine, S., Carbonnelle, E. \& Pelicic, V. Systematic functional analysis reveals that a set of seven genes is involved in fine-tuning of the multiple functions mediated by type IV pili in Neisseria meningitidis. Infect. Immun. 78, 3053-3063 (2010).

15. Stephens, D.S., Whitney, A.M., Rothbard, J. \& Schoolnik, G.K. Pili of Neisseria meningitidis. Analysis of structure and investigation of structural and antigenic relationships to gonococcal pili. J. Exp. Med. 161, 1539-1553 (1985).

16. Virji, M. et al. Pilus-facilitated adherence of Neisseria meningitidis to human epithelial and endothelial cells: modulation of adherence phenotype occurs concurrently with changes in primary amino acid sequence and the glycosylation status of pilin. Mol. Microbiol. 10, 1013-1028 (1993).

17. Nassif, X. et al. Roles of pilin and PilC in adhesion of Neisseria meningitidis to human epithelial and endothelial cells. Proc. Natl. Acad. Sci. USA 91, 3769-3773 (1994).

18. Winther-Larsen, H.C. et al. Neisseria gonorrhoeae PilV, a type IV pilus-associated protein essential to human epithelial cell adherence. Proc. Natl. Acad. Sci. USA 98, 15276-15281 (2001).

19. Lécuyer, H., Nassif, X. \& Coureuil, M. Two strikingly different signaling pathways are induced by meningococcal type IV pili on endothelial and epithelial cells. Infect. Immun. 80, 175-186 (2012).

20. Iacono, K.T., Brown, A.L., Greene, M.I. \& Saouaf, S.J. CD147 immunoglobulin superfamily receptor function and role in pathology. Exp. Mol. Pathol. 83, 283-295 (2007).

21. Sameshima, T. et al. Expression of EMMPRIN (CD147), a cell surface inducer of matrix metalloproteinases, in normal human brain and gliomas. Int. J. Cancer $\mathbf{8 8}$, 21-27 (2000).

22. Bertossi, M. et al. Developmental changes of HT7 expression in the microvessels of the chick embryo brain. Anat. Embryol. (Berl.) 205, 229-233 (2002).

23. Kimberley, F.C., Sivasankar, B. \& Paul Morgan, B. Alternative roles for CD59. Mol. Immunol. 44, 73-81 (2007).

24. Weksler, B., Romero, I.A. \& Couraud, P.O. The hCMEC/D3 cell line as a model of the human blood brain barrier. Fluids Barriers CNS 10, 16 (2013).

25. Schweitzer, K.M. et al. Characterization of a newly established human bone marrow endothelial cell line: distinct adhesive properties for hematopoietic progenitors compared with human umbilical vein endothelial cells. Lab. Invest. 76, 25-36 (1997).

26. Chen, Y. et al. Upregulation of HAb18G/CD147 in activated human umbilical vein endothelial cells enhances the angiogenesis. Cancer Lett. 278, 113-121 (2009).

27. Yu, X.L. et al. Crystal structure of HAb18G/CD147: implications for immunoglobulin superfamily homophilic adhesion. J. Biol. Chem. 283, 1805618065 (2008).

28. Mairey, E. et al. Cerebral microcirculation shear stress levels determine Neisseria meningitidis attachment sites along the blood-brain barrier. J. Exp. Med. 203, 1939-1950 (2006)

29. Helm, R.A. \& Seifert, H.S. Frequency and rate of pilin antigenic variation of Neisseria meningitidis. J. Bacteriol. 192, 3822-3823 (2010).

30. Lancellotti, M. et al. Conserved virulence of C to B capsule switched Neisseria meningitidis clinical isolates belonging to ET-37/ST-11 clonal complex. Microbes Infect. 8, 191-196 (2006).

31. Yurchenko, V., Constant, S., Eisenmesser, E. \& Bukrinsky, M. Cyclophilin-CD147 interactions: a new target for anti-inflammatory therapeutics. Clin. Exp. Immunol. 160, 305-317 (2010).

32. Join-Lambert, 0 . et al. Meningococcal interaction to microvasculature triggers the tissular lesions of purpura fulminans. J. Infect. Dis. 208, 1590-1597 (2013).

33. Melican, K., Michea Veloso, P., Martin, T., Bruneval, P. \& Dumenil, G. Adhesion of Neisseria meningitidis to dermal vessels leads to local vascular damage and purpura in a humanized mouse model. PLoS Pathog. 9, e1003139 (2013).

34. Gray, F. \& Alonso, J.M. in Greenfield's Neuropathology 6th edn. (eds. Graham, D.I. \& Lantos, P.L.) Ch. 3, 151-189 (2002).

35. Trust, T.J., Gillespie, R.M., Bhatti, A.R. \& White, L.A. Differences in the adhesive properties of Neisseria meningitidis for human buccal epithelial cells and erythrocytes. Infect. Immun. 41, 106-113 (1983).

36. Pinner, R.W., Spellman, P.A. \& Stephens, D.S. Evidence for functionally distinct pili expressed by Neisseria meningitidis. Infect. Immun. 59, 3169-3175 (1991).

37. Chen, Z. et al. Function of HAb18G/CD147 in invasion of host cells by severe acute respiratory syndrome coronavirus. J. Infect. Dis. 191, 755-760 (2005).

38. Watanabe, A. et al. CD147/EMMPRIN acts as a functional entry receptor for measles virus on epithelial cells. J. Virol. 84, 4183-4193 (2010).

39. Till, A. et al. A role for membrane-bound CD147 in NOD2-mediated recognition of bacterial cytoinvasion. J. Cell Sci. 121, 487-495 (2008).

40. Gabison, E.E., Hoang-Xuan, T., Mauviel, A. \& Menashi, S. EMMPRIN/CD147, an MMP modulator in cancer, development and tissue repair. Biochimie 87, 361-368 (2005).

41. Crosnier, C et al. Basigin is a receptor essential for erythrocyte invasion by Plasmodium falciparum. Nature 480, 534-537 (2011).

42. Harrison, T. et al. Erythrocyte $G$ protein-coupled receptor signaling in malarial infection. Science 301, 1734-1736 (2003). 


\section{ONLINE METHODS}

Antibodies and reagents. Anti-hCD147 mAb (clones MEM-M6/1 and MEM-M6/6) were purchased from AbD Serotec, anti-hCD59 mAb (clone MEM 43/5) from Tebu-Bio, anti-hCD44 mAb (clone J173) from Immunotech, anti-hICAM-1 mAb (clone 18C11) from R\&D Systems, anti-human collagen IV (clone PHM12) from Abcam, and anti-GFAP (clone G-A-5) and antivimentin (clone V9) from Sigma-Aldrich. Polyclonal antiserum raised against ezrin was obtained from P. Mangeat. mAb raised against PilE (20D9) and polyclonal antiserum raised against meningococcal $2 \mathrm{C} 4.3$ strain were described previously ${ }^{9}$. Secondary antibodies used for immunofluorescence labeling, chromogenic immunohistochemistry and immunoblotting were from Jackson ImmunoResearch Laboratories. Soluble chimeric CD147-Fc-His, ICAM-1-Fc and ALCAM-1-Fc-His molecules were purchased from R\&D Systems. DAPI, rhodamine-phalloidin, TPA, actinomycin D, isoproterenol, $\mathrm{DAB}$ peroxidase substrate and NBT/BCIP phosphatase alkaline substrate were purchased from Sigma-Aldrich.

Bacterial strains. $\mathrm{Nm} 2 \mathrm{C} 4.3$ (formerly clone 12 ) is a piliated capsulated $\mathrm{Opa}^{-}$ $\mathrm{Opc}^{-}$variant of the serogroup $\mathrm{C}$ meningococcal clinical isolate 8013 (ref. 43) cultured as described previously ${ }^{44}$. Nm2C4.3 expressing pilin variants $\mathrm{PilE}_{\mathrm{SA}}$, $\mathrm{PilE}_{\mathrm{SB}}$ or GFP were previously described ${ }^{28,43}$. The pilV mutant was engineered by introduction of an $a p h 3^{\prime}$ resistance cassette into the pilV gene. To complement the pilV mutant, the pilV allele was amplified using primers PilV forward $5^{\prime}$-CCT TAATTAAGGAGTAATTTTATGATGAGTAATAAAATGGAACA- ${ }^{\prime}$ ' and PilV reverse $5^{\prime}$-CCTTAATTAACTATTTTTTACGATTAGAGAAAGC-3', containing overhangs with restriction sites for PacI. This PCR fragment was restricted with $P a c I$ and cloned into PacI-restricted pGCC4 vector as described before ${ }^{12}$. This placed pilV under the transcriptional control of an IPTG inducible promoter within a DNA fragment corresponding to an intragenic region of the meningococcal chromosome. This pilV construct was then introduced into the chromosome of the $\Delta p i l V$ mutant by homologous recombination. A similar scheme was used for the construction of a complemented PilE isogenic meningococcus strain. The orf of the pilE gene was inserted, in E. coli, at the PacI restriction site of the PGCC4 plasmid. The primers used to amplify PilE were PilE forward 5'-CTTAATTAAGGAGTAATTTATGAACACCCTTCAAAAAGGTTTTAC-3' and PilE reverse $5^{\prime}$-CTTAATTAATTAGCTGGCAGATGAATCATCGCG-3'. The inducible pilE construct was inserted ectopically into the chromosome of the Nm2C4.3 strain through transformation, using erythromycin for selection. The wild-type pilE locus was subsequently inactivated through transformation of chromosomal DNA of a previously described aphA3-insertionally inactivated endogenous $\Delta$ pilE derivative and selected with kanamycin ${ }^{17}$. Virtually no PilE could be detected in the absence of induction in the resulting $\Delta$ pilE/pilE strain, whereas induction yielded up to approximately $5 \%$ of the wild-type expression level of PilE, as PilE is one of the most strongly constitutively expressed meningococcal proteins. Phenotypically, despite the low level of PilE complementation induced upon IPTG treatment, the functionality of type IV pili expressed upon PilE complementation was ascertained through restoration of competence (competence frequency $<1 \times 10^{-7}, 1 \times 10^{-5}$ and $1 \times 10^{-4}$ per $\mu \mathrm{g}$ DNA for $\Delta$ pilE, $\Delta$ pilE/ pilE and wild-type strains, respectively). Z5463 (formerly C396) is a capsulated, piliated $\mathrm{OpaA}^{+} \mathrm{Opc}^{+}$meningococcal clinical isolate of serogroup A (ref. 45). MC58 is a capsulated, piliated, $\mathrm{Opa}^{-} \mathrm{Opc}^{-}$strain of serogroup B ${ }^{46}$. FAM20 is a capsulated, piliated, $\mathrm{Opa}^{-} \mathrm{Opc}^{-}$strain of serogroup C (ref. 47). ROU is a capsulated piliated strain of serogroup W135 (ref. 48).

Cell lines. hCMEC/D3 is a fully differentiated human brain endothelial cell line derived from brain capillaries, produced in the laboratory and which recapitulates the major phenotypic features of the blood-brain barrier ${ }^{24}$. HBMEC is a human endothelial cell line isolated from bone marrow capillaries, provided by $\mathrm{B}$. Weksler, that maintains in vitro most of the characteristics of primary endothelial cells ${ }^{24}$. BB19 is a human brain endothelial cell line transformed by papilloma virus, provided by J.A. Nelson, which has lost most of the phenotypic features of brain endothelial cells ${ }^{49}$.

Cell culture, transfection and infection. To induce bacterial adhesion, BB19 cells were treated with the phorbol ester 12-O-tetradecanoylphorbol-13-acetate (TPA) $10 \mathrm{ng} \mathrm{ml}^{-1}$, for $10 \mathrm{~min}$, washed and bacterial adhesion measured $48 \mathrm{~h}$ after treatment. When indicated, $2 \mathrm{~h}$ after TPA treatment, the transcriptional inhibitor actinomycin D was added $\left(1 \mu \mathrm{g} \mathrm{ml}^{-1}\right.$, for $\left.4 \mathrm{~h}\right)$, cells were washed and adhesion was measured $42 \mathrm{~h}$ later. The hCMEC/D3 and HBMEC cell lines were cultured, transfected and infected as previously described ${ }^{7,9}$. Plasmid encoding human CD147 was provided by M. Bukrinsky, and plasmid encoding $\beta 2$ adrenergic receptor fused to YFP ( $\beta_{2}$-AR-YFP) was described previously ${ }^{50}$. To silence the expression of CD147, CD59, CD44, ICAM- 1 and $\beta_{2}$-AR, pools of four siRNA duplexes (ON-TARGET plus SMARTpool siRNA from Dharmacon) were used. The siControl siRNA (Dharmacon) was used as control. $48 \mathrm{~h}$ after transfection, efficiency of knockdown was assessed by FACS analysis or by reverse-PCR analysis for $\beta_{2}$-AR depletion as previously described ${ }^{9}$. When indicated, HBMEC cells were transfected with siRNA targeting the $3^{\prime}$ untranslated region of CD147, 3'-GCUGUCUGGUUGCGCCAUUUU-5' or control siRNA $3^{\prime}$-AUGUAUUGGCCUGUAUAG-5' (Eurogentech), and $40 \mathrm{~h}$ later, cells were again either mock transfected or transfected with CD147 cDNA coding region. $8 \mathrm{~h}$ later, surface expression of CD147 was quantified by FACS analysis and cells were infected concurrently with $\mathrm{Nm} 2 \mathrm{C} 4.3$.

Serial analysis of gene expression adapted for downsized extracts. $200 \mu \mathrm{g}$ of RNA extracted from $2 \times 10^{7}$ untreated or TPA-treated BB19 cells $16 \mathrm{~h}$ after treatment with TPA were used as a substrate for the serial analysis of gene expression adapted for downsized extracts (SADE) screen carried out as described ${ }^{51}$. Briefly, total RNA was extracted from cells, polyadenylated RNA was selected on oligo-dT columns and tagged cDNA was synthesized from the poly-A RNAs. Concatemers of DNA tags were sequenced, and the number of sequenced tags differentially represented in the two libraries was determined and analyzed using Monte Carlo statistical analysis.

Adhesion assays under static and flow conditions. Meningococcal adhesion on hCMEC/D3, HBMEC and BB19 cells was assayed under both static and shear stress conditions, as previously described ${ }^{9,28,52}$. When indicated, meningococci were preincubated with $5 \mu \mathrm{g} \mathrm{ml}^{-1}$ soluble recombinant human CD147-Fc and ICAM-1-Fc chimera before interaction with HBMEC cells with additional $2 \mu \mathrm{g} \mathrm{ml}^{-1}$ soluble proteins. Meningococcal adhesion was quantified following a 30-min infection in static conditions. To address the inhibitory effect of antibodies, HBMEC or hCMEC/D3 cells were grown on IBIDI chambers, pretreated for $1 \mathrm{~h}$ with $10 \mu \mathrm{g} \mathrm{ml}^{-1}$ antibodies targeting ICAM-1 (11C81) or CD147 (MEM-M6/1 and MEM-M6/6) and submitted to laminar flow $\left(0.04\right.$ dynes $/ \mathrm{cm}^{2}$ ) under an inverted microscope. GFP-expressing bacteria were introduced in the chamber under flow for $10 \mathrm{~min}$, and the number of adherent bacteria was determined, as previously described ${ }^{28}$. For adhesion assays on immobilized proteins, CD147-Fc and ICAM-1-Fc chimera were immobilized on glass slides using a modification of the technique described ${ }^{53}$. Briefly, slides were coated with $0.1 \%$ poly-L-lysine, washed with PBS, crosslinked with glutaraldehyde (0.5\%), washed, incubated with anti-Fc in assay buffer (PBS with 3\% BSA) for $2 \mathrm{~h}$, washed in assay buffer and incubated overnight at $4{ }^{\circ} \mathrm{C}$ with $2 \mu \mathrm{g} \mathrm{m}{ }^{-1}$ chimeric protein. Slides were washed before infection with meningococcal suspension of $\mathrm{OD}_{600}$ 0.05. After incubation with bacteria, slides were washed three times and fixed. Bacteria were labeled and visualized with a Leica DMI6000 microscope using a $63 \times$ oil-immersion objective. The number of adherent bacteria per field was quantified for 30 fields using ImageJ software.

Expression, purification and immobilization of maltose-binding proteinpilin recombinant proteins. Purified pilins were produced as fusion proteins with the maltose-binding protein (MBP), as described before ${ }^{9}$. Fragments of PilE ( $\mathrm{SA}$ and $\mathrm{SB}$ ), PilV, PilX and ComP lacking the region coding for amino acid residues 1 to 28 of the full-length proteins fused to the MBP were produced in E. coli, purified on amylose resin (New England BioLabs) and immobilized on Staphylococcus aureus (ATCC 25923) expressing specific receptors for the $\mathrm{Fc}$ domain of IgG as described before ${ }^{9}$. Coated bacteria were incubated with $1 \mu \mathrm{g}$ of CD147-Fc chimera for $30 \mathrm{~min}$ on ice. After 3 washes, bacteria were lysed with Laemmli buffer, and the quantity of coprecipitated CD147-Fc protein was assessed by immunoblot analysis. Bound CD147-Fc was quantified using ImageJ software. When indicated, hCMEC/D3 cells were pretreated with $10 \mu \mathrm{g} \mathrm{ml}^{-1}$ of MBP-pilins for $15 \mathrm{~min}$ before infection with meningococcal suspension of $\mathrm{OD}_{600} 0.05$ for $30 \mathrm{~min}$. After incubation with bacteria, cells were 
washed three times and the number of adherent bacteria determined as described above.

AlphaScreen. The AlphaScreen technology was used to assess the interaction between MBP-pilin recombinant fusion proteins and CD147-Fc-His or ALCAM-1-Fc-His as a control. The binding reaction was performed using white 384-well Optiplates (PerkinElmer, Whalham, MA, USA) in $20 \mu \mathrm{l}$ (total reaction volume). The AlphaScreen reagents (anti-MBP-coated Acceptor beads and Nickel chelate-coated Donor beads) were obtained from PerkinElmer. CD147Fc-His (or ALCAM-1-Fc-His) and MBP-pilins were prepared in $20 \mathrm{mM}$ Tris, $\mathrm{pH} 7.4,20 \mathrm{mM} \mathrm{NaCl}$. Donor beads $(20 \mu \mathrm{g} / \mathrm{ml})$ were incubated with CD147-His $(0,100$ or $500 \mathrm{nM})$ for $45 \mathrm{~min}$ at room temperature. In parallel, acceptor beads $\left(20 \mu \mathrm{g} \mathrm{m}^{-1}\right)$ were incubated with MBP-pilins $(0,50$ or $500 \mathrm{nM})$ for $1 \mathrm{~h}$ at room temperature in the AlphaScreen reaction buffer $\left(25 \mathrm{mM}\right.$ Tris, $\mathrm{pH} 7.4$ at $20^{\circ} \mathrm{C}$, $20 \mathrm{mM} \mathrm{NaCl}, 0.1 \%$ BSA and $0.05 \%$ Tween 20 ). Next, $10 \mu \mathrm{l}$ of each of the interacting partners were added to the plate, allowed to incubate for either $2 \mathrm{~h}$ or overnight in the dark and at room temperature. In antibody competition assays, $5 \mu \mathrm{l}$ of MEM-M-6/6 or 11C81 antibodies (at variable concentrations) were added to CD147-Fc-His-Donor beads for $30 \mathrm{~min}$ at room temperature before incubation with the MBP-pilin-Acceptor beads. Light signal was detected with the EnVision multilabel plate reader (PerkinElmer).

Surface plasmon resonance. Surface plasmon resonance (SPR) experiments were carried out on a Biacore 3000 SPR biosensor (Biacore InternationalAB). CD147-Fc or control ICAM-1-Fc chimera $\left(10 \mu \mathrm{g} \mathrm{ml}^{-1}\right)$ were immobilized onto CM5 chip surfaces at $10 \mu \mathrm{min}^{-1}$ using a standard amine coupling protocol with EDC (1-ethyl-3(3-dimethylaminopropyl) carbodiimide)/NHS ( $N$-hydroxysuccinimide) following the manufacturer's instructions. The density was controlled at an increased response level of 4,600 and 7,200 response units (RU) for ICAM-1-Fc and CD147-Fc, respectively. Soluble monomeric pilins diluted at various concentrations $\left(0.15\right.$ to $\left.10 \mu \mathrm{g} \mathrm{ml}^{-1}\right)$ in $10 \mathrm{mM}$ HEPES $\mathrm{pH}$ 7.4, $4 \mathrm{mM}$ EDTA, $0.005 \%(\mathrm{v} / \mathrm{v})$ P20 surfactant or $70 \mu \mathrm{l}$ of a suspension of pilin-coated staphylococci in PBS pH 7.4 were used as analytes. Analytes were applied at a flow rate of $10 \mu 1 \mathrm{~min}^{-1}$ for $5 \mathrm{~min}$ followed by a dissociation time of $6 \mathrm{~min}$. Regeneration of the sensorchip surface was performed with $2.5 \mathrm{M} \mathrm{NaCl}$ in PBS at $100 \mu \mathrm{lmin}^{-1}$ for $5 \mathrm{~min}$ followed by two washes at $100 \mu \mathrm{lmin}^{-1}$ with PBS. Output sensorgrams were analyzed using BIAcore BIAnalysis software.

Atomic force microscopy. Force spectroscopy was performed between pilins and a CD147-functionalized surface. CD147-Fc-His or control ALCAM-1Fc-His chimera were deposited on a glass surface using a nitrilotriacetic acid that ensured covalent and oriented binding with the protein ${ }^{54}$. MBP-pilins and control proteins (MBP, cyclophilin) were coated on AFM tips using established protocol known to keep protein functionality intact as previously published ${ }^{55,56}$. All the experiments were performed at room temperature on a commercial AFM (MultiMode8, Bruker, Santa Barbara, CA) driven under Nanoscope 8.15. MBP-pilins were used at $100 \mu \mathrm{g} \mathrm{ml}^{-1}$. Bruker DNP cantilevers with a nominal spring constant of $0.06 \mathrm{~N} / \mathrm{m}$ and a nominal tip radius of $20 \mathrm{~nm}$ were used. True cantilever spring constant was determined using Bruker's thermal tune calibration tool. Experiments were done in force-volume mode operating in $150 \mathrm{mM}$ $\mathrm{NaCl}, 20 \mathrm{mM}$ HEPES buffer ( $\mathrm{pH}$ 7.0). Pulling speed was set to $10 \mu \mathrm{m} \mathrm{s}^{-1}$ on $4 \mu \mathrm{m}^{2}$ areas, $300 \mathrm{~nm} \mathrm{z}$ ramp, with a relative trigger of $150 \mathrm{pN}$ on the cantilever deflection and a contact time of $200 \mathrm{~ms}$. 6,144 force curves per condition were recorded, using at least 6 different tips. Most nonspecific tip-surface adhesion events were automatically discarded from analysis. Force-distance curves were analyzed as previously described ${ }^{55}$, and binding-unbinding events on the retraction curve were detected according to their shape and size characteristics by a fuzzy logic algorithm fully described in (ref. 57). Briefly, we considered unbinding events based on the vertical segment size, the V-shape and the angle with the baseline. Statistical analysis was performed using R2.15 where provided data are the peak position mean values \pm s.d.

Confocal immunofluorescence microscopy. hCMEC/D3, BB19 or HBMEC cells were grown to confluence on Permanox coverslips (Thermo Fisher Scientific) or on Transwell filters (Costar). After the indicated treatments and/or infection, cells were fixed and labeled as previously described ${ }^{44,52}$. Anti-ezrin antibody (1/1,000), anti-CD44 (1/25) anti-CD147 (MEM-M6/1, 1/100), antiCD59 (1/100) and rabbit polyclonal serum anti-Nm2C4.3 strain $(1 / 3,000)$ were used as primary antibodies, and DAPI $\left(0.5 \mathrm{mg} \mathrm{ml}^{-1}\right)$ was added to Alexa Fluor-conjugated secondary antibodies (1/300). Image acquisition and analysis were performed with a DMI6000 microscope (Leica, 63×). Series of optical sections were obtained with a confocal spinning disk microscope (Leica, 63×). Three-dimensional (3D) reconstructions were obtained using Imaris software and quantification histograms with ImageJ software (NIH). Quantitative analysis of protein recruitment under bacterial colonies was determined as the proportion of colonies positive for the protein of interest indicated. At least 50 colonies were observed per coverslip. Each experiment was repeated at least 3 times in duplicate or triplicate.

Infection of severe combined immunodeficiency mice grafted with human skin. Six-week-old CB17/Icr-Prkdscid/IcrIcoCrl SCID female mice were purchased from Charles River Laboratories (Saint Germain sur l'Arbresle, France). Human skin tissues were obtained from adult patients undergoing plastic surgery in the Service de Chirurchie Plastique et Reconstructive of the Groupe Hospitalier Saint-Joseph (Paris, France). In accordance with the French legislation, patients were informed and did not refuse to participate in the study. Experimental procedures were performed as previously described ${ }^{32}$, in accordance with the guidelines of the Institut National de la Santé et de la Recherche Médicale. The use of human tissue and the experimental protocol was approved by the Animal Experimentation Ethics Committee of the Université Paris Descartes (consent form CEEA34.O.J.L.039.12). Briefly, mice were prepared for transplantation by shaving the hair of the back and abdominal areas after they received an intraperitoneal injection of ketamine $100 \mathrm{mg} \mathrm{kg}^{-1}$ and xylazine $10 \mathrm{mg} \mathrm{kg}-1$. A skin flap was created, and a full-thickness human skin graft was placed onto the wound bed. The transplants were held in place with 6-0 nonabsorbable monofilament suture materials, and the flap was then sutured above the transplant. Grafted mice were used for meningococcal infection experiments 1 month after human skin transplantation. Meningococcus strains were grown overnight at $37^{\circ} \mathrm{C}$ on GCB agar plates prepared without iron and supplemented with $15 \mu \mathrm{M}$ deferoxamine (Desferal, Novartis) with appropriate antibiotic. Bacterial colonies were harvested and cultured in RPMI with $1 \%$ bovine serum albumin medium and $0.06 \mu \mathrm{M}$ deferoxamine with gentle agitation to reach the exponential phase of growth. Bacteria were then resuspended in physiological saline containing $20 \mathrm{mg} \mathrm{ml}^{-1}$ of human holotransferrin to promote bacterial growth in vivo (2914HT, $\mathrm{R} \& \mathrm{D}$ systems). Mice were infected intraperitoneally with $0.5 \mathrm{ml}$ of this bacterial suspension to minimize the inoculum required to obtain a reproducible high bacteremia in grafted mice. To assess bacteremia in infected animals, $10 \mu \mathrm{l}$ of blood was sampled using a heparinized hematocrit glass tube $1 \mathrm{~h}$ after injection by puncture in the lateral tail vein, or $18 \mathrm{~h}$ after injection by intracardiac puncture on animal killed by intraperitoneal injection of a lethal dose of ketamine and xylazine. Bacterial counts were performed by plating serial dilutions of blood on GCB agar plates. Results were expressed in colony-forming units (CFU) per $\mathrm{ml}$ of blood. Biopsy of human skin grafts were carefully collected using a dermatological punch biopsy system and fixed overnight at $4{ }^{\circ} \mathrm{C}$ in paraformaldehyde $1 \%$, lysin $75 \mathrm{mM}$ and sodium periodate $100 \mathrm{mM}$ in phosphate buffer. After they were washed in phosphate buffer, the specimens were dehydrated in successive sucrose gradient solutions $(10 \%, 20 \%, 30 \%$ prepared in phosphate buffer $0.1 \mathrm{M})$ for $2 \mathrm{~h}$ at $4{ }^{\circ} \mathrm{C}$ each, embedded in OCT and then frozen at $-80^{\circ} \mathrm{C} .7-\mu \mathrm{m}$ thick sections of the dermis were immobilized on Superfrost plus microscope slides and analyzed via immunofluorescence. Sections were incubated with the following primary antibodies for $2 \mathrm{~h}$ in PBS/BSA $0.1 \%$ : monoclonal anti-human collagen IV (1/100) and a rabbit polyclonal serum against the Nm2C4.3 strain $(1: 3,000)$. DAPI $\left(0.5 \mathrm{mg} \mathrm{ml}^{-1}\right)$ was added to Alexa Fluor-conjugated secondary antibodies (1/300) for $1 \mathrm{~h}$. After additional washing, coverslips were mounted in glycergel (Dako). 3 sections per graft were analyzed using epifluorescence (Zeiss Axiovert, $20 \times$ ). Quantification analysis of the images ( $n>10$ per graft) was performed using ImageJ software (NIH). Results are presented as a vascular colonization index corresponding to the area occupied by the fluorescently labeled bacteria in relation to the human vessel area delineated by the anticollagen IV staining $\times 100$, from 6 independent grafts per condition. 
Infection of human brain tissues. Fresh human brain sections were obtained from frontal lobe specimens of macroscopically and histologically normal brain (confirmed by a neuropathologist) of individuals referred to the Department of Forensic Medicine for unexplained out-of-hospital sudden death (consent forms ML1094, PFS 10-008, ClinicalTrials.gov NCT00320099 approved by The Institutional Review Board of the Poincaré Hospital, Versailles-Saint Quentin University and the Agence de la Biomédecine). After freezing of the brain tissue with isopentane cooled in liquid nitrogen, the $7-\mu \mathrm{m}$-thick sections containing leptomeninges, cortical ribbon and the underlying white matter were immobilized on Superfrost plus microscope slides and stored at $-80^{\circ} \mathrm{C}$. Defrosted sections were rehydrated in PBS for $5 \mathrm{~min}$ and incubated for $1 \mathrm{~h}$ with medium containing $0.1 \%$ BSA before infection with suspensions of bacteria $\left(2 \times 10^{7}\right.$ in $150 \mu \mathrm{l}$ of medium containing $0.1 \% \mathrm{BSA}$ ) for $1 \mathrm{~h}$ at $37^{\circ} \mathrm{C}$. Sections were then gently washed horizontally 5 times and fixed in PAF $4 \%$ for $10 \mathrm{~min}$ at room temperature. When indicated, sections were treated for $1 \mathrm{~h}$ with $10 \mu \mathrm{g} \mathrm{ml}^{-1}$ of antibodies targeting CD147 or ICAM-1 and then washed 3 times before infection by bacteria. Adherent meningococci were detected by chromogenic immunohistochemistry and immunofluorescence analysis. To perform chromogenic immunohistochemistry, after tissue rehydration, sections were incubated overnight with the indicated primary antibodies (monoclonal anti-human CD147 (MEM-M6/1, 1/100) and a rabbit polyclonal serum anti-Nm2C4.3 strain $(1 / 3,000))$ and revealed using horseradish peroxidase-coupled secondary donkey anti-mouse antibody and an alkaline phosphatase donkey anti-rabbit (1/500). $\mathrm{DAB}$ (brown color) and NBT/BCIP (blue color) were used as chromogens. For immunofluorescence analysis, brain sections were incubated with the following primary antibodies for $2 \mathrm{~h}$ in PBS/BSA 0.1\%: monoclonal anti-human CD147 (MEM-M6/1, 1/100), anti-GFAP (1/100), anti-human vimentin (1/100) and a rabbit polyclonal serum anti-Nm2C4.3 strain $(1 / 3,000)$. Alexa Fluor-conjugated phalloidin $(1 / 300)$ and DAPI $\left(0.5 \mathrm{mg} \mathrm{ml}^{-1}\right)$ were added to Alexa Fluorconjugated secondary antibodies (1/300) for $1 \mathrm{~h}$. After additional washing, coverslips were mounted in glycergel (Dako). Entire samples were scanned using Nanozoomer 2.0 (Hamamatsu) and were further analyzed using optical microscopy, epifluorescence (Zeiss Axiovert, 40x) and confocal (spinning disk Leica, $63 \times)$ microscopy. Quantification analysis of the fluorescently labeled bacteria that adhered on a $1 \mathrm{~mm}^{2}$ surface area was performed using ImageJ software. Results are presented as a mean of fluorescence per $100 \mu \mathrm{m}^{2}$, from two independent experiments. 3D reconstructions were performed on deconvoluted confocal stacks using Imaris software.
Statistical analyses. All values are expressed as the mean \pm s.e.m. Statistical differences were determined to be significant at $P<0.05$. The specific tests used are described in the figure legends. All analyses were performed using GraphPad Prism software. No statistical method was used to predetermine sample size. The investigators were not blinded to allocation during experiments and outcome assessment. The experiments were not randomized.

43. Nassif, X. et al. Antigenic variation of pilin regulates adhesion of Neisseria meningitidis to human epithelial cells. Mol. Microbiol. 8, 719-725 (1993).

44. Hoffmann, I., Eugene, E., Nassif, X., Couraud, P.O. \& Bourdoulous, S. Activation of ErbB2 receptor tyrosine kinase supports invasion of endothelial cells by Neisseria meningitidis. J. Cell Biol. 155, 133-143 (2001).

45. Achtman, M. et al. Purification and characterization of eight class 5 outer membrane protein variants from a clone of Neisseria meningitidis serogroup A. J. Exp. Med. 168, 507-525 (1988).

46. Tettelin, $\mathrm{H}$. et al. Complete genome sequence of Neisseria meningitidis serogroup B strain MC58. Science 287, 1809-1815 (2000).

47. Dyer, D.W., McKenna, W., Woods, J.P. \& Sparling, P.F. Isolation by streptonigrin enrichment and characterization of a transferrin-specific iron uptake mutant of Neisseria meningitidis. Microb. Pathog. 3, 351-363 (1987).

48. Pron, B. et al. Interaction of Neisseria meningitidis with the components of the blood- brain barrier correlates with an increased expression of PilC. J. Infect. Dis. 176, 1285-1292 (1997).

49. Prudhomme, J.G. et al. Studies of Plasmodium falciparum cytoadherence using immortalized human brain capillary endothelial cells. Int. J. Parasitol. 26, 647-655 (1996).

50. Angers, S. et al. Detection of $\beta$ 2-adrenergic receptor dimerization in living cells using bioluminescence resonance energy transfer (BRET). Proc. Natl. Acad. Sci. USA 97, 3684-3689 (2000).

51. Virlon, B. et al. Serial microanalysis of renal transcriptomes. Proc. Natl. Acad. Sci. USA 96, 15286-15291 (1999).

52. Lambotin, M. et al. Invasion of endothelial cells by Neisseria meningitidis requires cortactin recruitment by a PI3-kinase/Racl signalling pathway triggered by the lipo-oligosaccharide. J. Cell Sci. 118, 3805-3816 (2005).

53. Steffen, A. et al. MT1-MMP-dependent invasion is regulated by TI-VAMP/VAMP7. Curr. Biol. 18, 926-931 (2008).

54. Chevalier, S. et al. Creating biomimetic surfaces through covalent and oriented binding of proteins. Langmuir 26, 14707-14715 (2010).

55. Roduit, C. et al. Elastic membrane heterogeneity of living cells revealed by stiff nanoscale membrane domains. Biophys. J. 94, 1521-1532 (2008).

56. Yersin, A. et al. Elastic properties of the cell surface and trafficking of single AMPA receptors in living hippocampal neurons. Biophys. J. 92, 4482-4489 (2007).

57. Kasas, S., Riederer, B.M., Catsicas, S., Cappella, B. \& Dietler, G. Fuzzy logic algorithm to extract specific interaction forces from atomic force microscopy data. Rev. Sci. Instrum. 71, 2082-2086 (2000). 